\title{
MODELING MULTI-AGENT SYSTEMS WITH HIERARCHICAL COLORED PETRI NETS
}

\author{
Bingxian $\mathrm{Ma}^{1,2}$ \\ '(Institute of Computing Technology, Chinese Academy of Sciences, Beijing, China, 100080 , \\ '(The Graduate School of the Chinese Academy of Sciences, Beijing, China, 100039)
}

\begin{abstract}
A new approach to model multi-agent systems with hierarchical colored Petri nets is introduced. Every agent has its colored Petri net system, when agents are composed into a MAS, we get hierarchical colored Petri net system of the multi-agent system, properties of the hierarchical colored Petri net system, such as reachability, deadlock detection and avoidance, fairness can be used to analyze dynamic properties of the multi-agent system.
\end{abstract}

Key words: agent, multi-agent system, hierarchical colored Petri nets

\section{INTRODUCTION}

Multi-agent systems (MASs, [1,2]) provide robust and scalable approach for the solution of complex problems. In a MAS, different agents are organized working together to achieve the project, Each individual agent is organized to be a specialist for a particular task of the MAS, but none has the capability of performing all the tasks required of the project. Agents will cooperate and interact with others to achieve the project. MASs are obviously dynamic systems, composing heterogeneous agents running to achieve users demands. The technologies of modeling and analyzing of MAS are very important for the using of agents technology, which is not a new idea. Many works $([2,3,4,5,6])$ have been done to model and analysis the inter-relationships among agents, while their methods are not good at modeling the distributed and concurrent events in a MAS at the system level. 
Petri nets $([7,8])$ are applicable to a variety of fields and systems, especially suitable for systems with distributed and concurrent events ,and have been used to model and analyze MASs. [9] firstly modeled single agent behaviors with Petri nets, [10]uses predicate/transition nets model and analysis multi-agent behaviors, a single $\operatorname{Pr} / \mathrm{T}$ net is used to model the whole multi-agent problem, especially multi-agent plans.[3] using system net, agent nets and connectors nets model the mobile agents environment, mobile agent and connectors. [11] use agents based G-net (a extended G-net([3])) model agents, which have explicit asynchronous communication interface and support formal reasoning of agents behaviors. Colored Petri nets([12]) ,combining the advantages of Petri nets and high level programming languages, are suit to model complex systems with concurrent and dynamic behaviors. $[4,5,6]$ use colored Petri nets model the sociality, conversation and interaction between agents in multi-agent systems. These works mostly are about just one aspect property of a MAS.

How to embed Petri nets technologies into the dynamic running processes and get full-sided analysis of a MAS are the problems that we are interested in. In this paper, we will set out a new approach to model MAS with hierarchical colored Petri nets. Each agent has its colored Petri net system, when composed into a MAS we can get its hierarchical colored Petri net system of the MAS, through which we are able to get the dynamic properties of the MAS, including qualitative and quantitative properties.

The remainder of this paper is structured as follows: In section 2 we briefly present related concepts and theories about this paper, in section 3, we model and analyze MAS with hierarchical colored Petri net system; we conclude current workings and present further work in section 4 .

\section{AGENT AND HIERARCHICAL COLORED PETRI NETS}

Agents are mostly used to denote a hardware or (more usually) softwarebased computer systems that enjoy the following properties $([1,2])$ : autonomy, social ability, reactivity, and pro-activeness. MAS is an approach for the solution of complex problems. In a MAS, different agents are organized work together to achieve the project, Each individual agent is organized to be a specialist for a particular task of the MAS, but none has the capability of performing all the tasks required of the project. Agents will cooperate and interact with others to achieve the project. For the smoothly running of the MAS, effective formal model and analysis methods and tools are essentially. 
Colored Petri nets (CP-nets, [12]) attaching a color to each token and a color set to each place allows us to use fewer places than would be needed in a PT-net without losing any properties. The basic idea behind Hierarchical Colored Petri nets (HCP-net) is to allow the modeler to construct a large model by combining a number of small CP-nets into a larger net which is similar to the situation in which a programmer constructs a large program from a set of modules and subroutines. It is always possible to translate a $\mathrm{HCP}$-net into a non hierarchical CP-net-which in turn can be translate into an ordinary Petri net, which means that the theoretical modeling powers of these three classes of Petri nets are same.

\section{MODELING MULTI-AGENT SYSTEMS WITH HIERARCHICAL COLORED PETRI NETS}

We set up a new approach to model MASs with HCP-nets technology. Firstly, the structure of an agent is based on BDI theories $([1,2])$ and its behaviors can be modeled with a non-hierarchical $\mathrm{CP}$-net, the structure of an agent is:

\begin{tabular}{|l|c|}
\hline \multicolumn{1}{|c|}{ Agent Profile } & $\begin{array}{c}\text { Description of the agent, name, } \\
\text { goals, intentions, beliefs. }\end{array}$ \\
\hline Agent Process Model & $\begin{array}{c}\text { Description of the agent how to } \\
\text { work, modeled with colored Petri } \\
\text { nets. }\end{array}$ \\
\hline Agent Information Table & $\begin{array}{c}\text { Registers agents that cooperate } \\
\text { with the agent recently. }\end{array}$ \\
\hline
\end{tabular}

The process model is a non-hierarchical CP-net that used to model agent's behavior logic mechanism, with which we can get the agent's states transformation and behaviors schedules to achieve a goal. Agent information tables will help we effectively organizing a dynamic MAS.

Secondly, MAS can be modeled with its HCP-net system, each agent within it is denoted as Figurel in the prime page and process models of agents are sub-pages of the HCP-net system. The MAS is described at system level and agent level. At system level, the structure of MAS is modeled with the prime page (a non-hierarchical CP-net), agents are presented with their substitution of transitions, we can get the relationships among agents and monitor the MAS from the running of its HCP-net system. At agent level, each agent is particular modeled in this level and we can get each agent's behaviors and states transitions to achieve its goals, It is just as the way that model a large-scale system at macro- and micro- levels with hierarchical modeling technologies. 
receiving interface sending interface

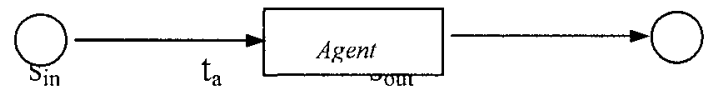

Figure1 Substitution transition of agent

Note: The HCP-net system of a MAS is bounded Petri nets system.

Because users would not waiting for a system running absoluteness long time without any outcome, when the system running longer than their expect time, they will abandon or adjust it.

The analysis of MAS can be transformed into its Petri net system properties analysis, including qualitative and quantitative properties, more than just partially about coordination or negotiation between agents([4,5,6]), such as:

1)Reachability: when MAS set out to deal with a goal, the system must reaches a state that denotes the goal had been achieved(goal state). From initial state to goal state, the system involving several states transformations from one to another. Markings of the HCP-net are able to exactly describe the system's states. $M_{0}$, the initial marking of the HCP-net corresponds with initial state of the MAS, and $M_{1}$ corresponds with the goal state. If $M_{1}$ can be reached from $M_{0}$, it means the goal can be achieve; else the goal can not be achieved and we should do some adjustment for the MAS.

2) Deadlock detection and avoidance. Through coordination, agents within MAS can work together to ensure coherent action. Conflict resolution is essential for coordinated agent behaviors. When the HCP-net system of MAS running into a deadlock, it means conflicts resolution is needed in the MAS. Reasons and resolution methods to deadlock states of the HCP-net system can be applied to resolve or avoid conflicts in the MAS.

3) Fairness. Fairness property describes the dependences among transitions in the HCP-net, accordingly, the dependence among agents in the MAS.

\section{CONCLUSION AND FURTHER WORK}

We have dealt with the approach to model MAS with its HCP-nets system. Each agent has its colored Petri net system and when agents are composed into a MAS, we get its HCP-net system. Properties of the HCP- 
net system, such as reachability, deadlock detection and avoidance, fairness, can be used to analyze dynamic properties of the MAS.

Our future work reminded to be done includes: a) dynamic organizing procedure of a MAS based on the agent structure in section 3; b) conflicts detection and avoiding in the MAS with Petri nets methods.

\section{REFERENCE}

[1] Wooldridge, M., and Jennings, N, "Intelligent agents: theory and practice", The Knowledge Engineering Review. 1995.

[2]Shi Zhongzhi, Intelligent agents and its Applications, Science Press, 2000(in Chinese)

[3] A.Perkusich and J.de Figueiredo, "G-net: A Petri Net Based Approach for Logicaal and Timing Analysis of Complex Software Systems" ,.Journal of systems and Software, 39(1):39-59,1997

14] Danny Weyns \& Tom Holvoet “ A Colored Petri Net for a Multi-Agent Application." .Aarhus, Denmark - August 27, 2002..MOCA '02.

[5] D.Moldt and F.Wienberg. "Multi-Agent-Systems based on Coloured Petri Nets" , Proceedings of the $18^{\text {th }}$ international Conference ICATPN'97, Toulouse, France, 1997.

[6] R. Cost et al., Modeling Agent Conversations with Colored Petri Nets, IJCAI '99, Stockholm, Sweden, 1999.

[7] T. Murata : "Petri nets: Properties, Analysis and Application" .Proceedings of the IEEE Vol.77 no.4(April 1989),541-580.

[8] Yuan Chongyi,Theories of Petri Nets, China Publishing House of Electronics Industry, 1998, (in Chinese)

[9] T. holvoet. "Agents and Petri nets." Petri net news letter, (49):3-8 Oct-1995

[10] D. Xu, R. A. Volz, T. R. Ioerger, and J. Yen, "Modeling and Verifying Multi-Agent BehaviorsUsing Predicate/Transition Nets," Proc. of the 14th International Conference on Software Engineering and Knowledge Engineering, Italy, July 2002.

[11] H. Xu and S. M. Shatz, "An Agent-based Petri Net Model with Application to Seller/Buyer Design in Electronic Commerce," ISADS 2001, March 2001, Dallas, Texas, USA.pp.11-18.

[12] Jensen,K.: Colored Petri Nets. Basic Concepts ,Analysis Methods and Practical Use. Volume 1,Basic Concepts. Monographs in Theoretical Computer Science .Berlin, Heidelberg, New York:Springer-Verlag, ${ }^{\text {nd }}$ corrected printing 1997,ISBN:3-540-60943-1 